\title{
INITIAL BOUNDARY VALUE PROBLEMS FOR INTEGRABLE SYSTEMS: TOWARDS THE LONG TIME ASYMPTOTICS
}

\author{
ANNE BOUTET DE MONVEL*, VLADIMIR P. KOTLYAROV ${ }^{\dagger}$, DMITRY SHEPELSKY $^{\dagger}$, \\ AND CHUNXIONG ZHENG ${ }^{\ddagger}$
}

\begin{abstract}
The long time behavior of solutions of (Dirichlet) initial boundary value problems for the focusing nonlinear Schrödinger equation in the case of (asymptotically) periodic boundary conditions of special (one-frequency) structure is considered both theoretically and numerically. The results of numerical simulations are shown to confirm the theoretical description in the parameter ranges where the assumption about the one-frequency character of the Neumann boundary values is (theoretically) admissible, whereas in the other ranges they suggest a more complicated description of the behavior of these values.
\end{abstract}

\section{INTRODUCTION}

The evaluation of the long time behavior of solutions of evolution nonlinear systems, continuous as well as discrete, is the most challenging problem in the theory of such system. The last 15 years have clearly shown that for integrable systems (in the sense that there exists an associated Lax pair), the appropriate tool is the inverse scattering transform method in the form of Riemann-Hilbert problems (RHP), the use of which in the study of long time behavior is performed in the framework of the nonlinear steepest descent method [12].

This approach allowed obtaining rigorous results concerning the long time behavior of solutions of Cauchy problems, i.e., initial value problems for various equations in dimension $1+1$ (1D space-type variable + time variable), where the initial data specified on the whole line of the space variable are rapidly decaying at infinity $[10,13]$. Recently, this method has been generalized to the study of asymptotics of Cauchy problems with non-vanishing initial data, with constant or periodic background [11].

The primary step of the RHP method for studying the long time behavior of solutions of well-posed initial value problems consists in representing the solutions in terms of the solution of an associated Riemann-Hilbert problem, all the data for which - the jump matrix and, possibly, residue conditions - are uniquely determined by the given initial data, in terms of the associated spectral functions. Then the study of the long time behavior of the solution of a nonlinear equation in question reduces to the study of the long time asymptotics of this solution of this Riemann-Hilbert problem. Usually, the jump matrix of the problem, as it appears naturally in its original setting, oscillates rapidly as $t \rightarrow \infty$ and thus a series of transformations is needed in order to reduce the problem to a "model problem" with

Date: July 17, 2008.

2000 Mathematics Subject Classification. Primary: 37K15, Secondary: 35Q55, 35Q15, 65M12.

Key words and phrases. initial boundary value problem, integrable system, focusing nonlinear Schrödinger equation, long time asymptotics, Riemann-Hilbert problem. 
decaying (to zero, or to constant matrices, possibly with different values on different parts of the contour) jump matrices, so that the resulting (model) problem can be solved explicitly.

\section{INITIAL-BOUNDARY VALUE PROBLEMS}

A natural problem arises: generalize this powerful method to the case of initial boundary value (IBV) problems, where, along with initial data, certain boundary conditions are specified for a well-posed problem. The simplest setting seems to be the following:

Problem 1. For a $1+1$ equation, find a solution in the quarter plane $x>0, t>0$ having prescribed

- initial data, for $t=0, x \in[0,+\infty)$, decaying as $x \rightarrow+\infty$,

- and certain boundary data, for $x=0, t \in[0,+\infty)$.

To fix ideas, consider the focusing nonlinear Schrödinger equation (NLS)

$$
\mathrm{i} q_{t}+q_{x x}+2|q|^{2} q=0, \quad x>0, t>0 .
$$

It is known that the Dirichlet boundary value problem is well-posed, if one specifies decaying (as $x \rightarrow+\infty$ ) initial values

$$
q(x, 0)=q_{0}(x), \quad x \in[0,+\infty)
$$

and boundary values

$$
q(0, t)=g_{0}(t), \quad t \in[0, T]
$$

for any $T>0$; for the precise statements concerning the well-posedness (the functional classes for boundary functions, the definitions for a solution of various (weak) types, etc.), see, e.g., $[17]$ and the references therein. Particularly, for any fixed $T$, sufficiently smooth data, $g_{0}(t)$, $t \in[0, T]$ and $q_{0}(x), x>0$ determine uniquely the classical solution $q(x, t)$ of $(2.1)$ in the half-strip $x>0, t \in[0, T]$.

A natural question is:

Question 1. What happens with this solution as $t \rightarrow+\infty$ ?

Evidently, the answer to this question depends crucially on the behavior of the boundary data $g_{0}(t)$ as $t \rightarrow+\infty$.

2.1. The RHP approach for IBV problems. A generalization of the RHP approach to the case of initial boundary value (IBV) problems was proposed by Fokas $[14,15]$ and was further developed for studying various nonlinear equations $[3,9,16]$. Similarly to the case of initial value problems, the key feature of the method is the representation of the solution to a nonlinear equation in terms of an associated RHP, the data for which are constructed in terms of values of the sought solution on the boundary of the domain in the $(x, t)$-plane, where the problem is posed, i.e., at $t=0$ and at $x=0$. However, there is a crucial difference: in the case of IBV problems, the spectral functions needed for the construction of the RHP are determined by an "excessive" number of boundary values. Particularly, in the case of the NLS equation, a part of the spectral functions needed for the construction of the RHP are determined in terms of $g_{0}(t)$ and $g_{1}(t)=q_{x}(0, t)$. The reason for this is that the $t$-operator in the associated Lax pair involves $q$ and $q_{x}$ in the construction of its coefficient matrix. 
Thus, in order to construct the RHP (which will serve as basis for the subsequent long time analysis), we have to specify, all together, $q_{0}(x), g_{0}(t)$, and $g_{1}(t)$. Clearly, one cannot prescribe these three functions (to be the boundary values of a certain solution of the NLS equation) arbitrarily: the well-posedness results show that if one specifies, say, $q_{0}(x)$ and $g_{0}(t)$, then $g_{1}(t)$ is uniquely determined by these data.

2.2. The Dirichlet-to-Neumann map. It turns out that the compatibility of the set of functions $\left\{q_{0}(x), g_{0}(t), g_{1}(t)\right\}$ can be characterized, in a rather simple form, in terms of the associated spectral data [16]. Combining this characterization with the Gelfand-LevitanMarchenko integral representation of solutions of the $t$-equation in the Lax pair allowed translating the compatibility into the form of a (nonlinear) equation characterizing the "nonlinear Dirichlet-to-Neumann map", i.e., the mapping [2]

$$
\left\{q_{0}, g_{0}\right\} \rightsquigarrow g_{1} \text {. }
$$

in the physical variables. Although this map turns out to be rather complicated, it has been successfully used, in terms of non-reflecting boundary conditions, in the numerical simulations of solutions of the initial value problem for the NLS equation with "almost" finite initial conditions [19].

Theoretically, this map can be used for the study of the long time behavior of $g_{1}(t)$, given $q_{0}(x)$ and $g_{0}(t)$ for all $t \in[0,+\infty)$, but at present, this remains an open problem.

On the other hand, in the implementation of the RHP method for the study of long time asymptotics, this information is crucially needed. Indeed, suppose that $g_{0}(t)$ is given for all $t \in[0,+\infty)$. Then it is the behavior of $g_{1}(t)$ as $t \rightarrow+\infty$ that determines the type of spectral problem for the $t$-equation (2.4b) in the Lax pair for the NLS equation

$$
\begin{aligned}
& \psi_{x}+\mathrm{i} k \sigma_{3} \psi=Q \psi, \\
& \psi_{t}+2 \mathrm{i} k^{2} \sigma_{3} \psi=\tilde{Q} \psi,
\end{aligned}
$$

where $\sigma_{3}=\operatorname{diag}(1,-1)$ and

$$
Q=\left(\begin{array}{cc}
0 & q \\
-\bar{q} & 0
\end{array}\right), \quad \tilde{Q}=\left(\begin{array}{cc}
\mathrm{i}|q|^{2} & 2 k q+\mathrm{i} q_{x} \\
-2 k \bar{q}+\mathrm{i} \bar{q}_{x} & -\mathrm{i}|q|^{2}
\end{array}\right) .
$$

Recall that in the RHP method for the NLS equation on the half-line $x>0$, the "master Riemann-Hilbert problem" is constructed from two sets of spectral functions:

- the first one appears as the spectral functions in the scattering problem for equation (2.4a), on the zero background, with the potential $q=q_{0}(x)$,

- whereas the second one consists of the spectral functions in the scattering problem for equation (2.4b), where $q=g_{0}(t)$ and $q_{x}=g_{1}(t)[16]$.

Under the assumption that $\left\{q_{0}(x), g_{0}(t), g_{1}(t)\right\}$ are compatible and that $g_{0}(t) \rightarrow 0$ and $g_{1}(t) \rightarrow$ 0 sufficiently fast as $t \rightarrow+\infty$, the solution $q(x, t)$ of the associated IBV problem for the NLS equation behaves, as $t \rightarrow+\infty$, roughly speaking, similarly to the case of the initial value problem: there can be solitons propagating along certain directions in the quadrant $x>0$, $t>0$, while the "rest" of the solution exhibits decaying (of order $\mathrm{O}\left(t^{-1 / 2}\right)$ ) modulated oscillations [16]. The soliton parameters, as well as the parameters of the oscillations, are determined, in the case of the IBV problem, in terms of spectral functions associated with both initial and boundary conditions. 
Then a natural question emerges.

Question 2. What one can see in the asymptotics if the boundary values are non-decaying as $t \rightarrow+\infty$ (assuming that the initial values $q_{0}(x)$ are still fast decaying as $\left.x \rightarrow+\infty\right)$ ?

\section{Periodic boundary Conditions}

The simplest non-decaying boundary conditions seem to be asymptotically periodic, with the simple exponential dependence on $t$ :

$$
q(0, t)=g_{0}(t)=a \mathrm{e}^{2 \mathrm{i} \omega t}+g_{0}^{(1)}(t)
$$

with some numbers $a>0$ and $\omega \in \mathbb{R}$, and with $g_{0}^{(1)}(t) \rightarrow 0$ fast enough as $t \rightarrow+\infty$.

3.1. $\boldsymbol{\omega}<-\mathbf{3 a}^{\mathbf{2}}$. In [6], the problem is addressed of constructing an associated RHP under assumption that

$$
\begin{aligned}
& q_{x}(0, t)=g_{1}(t)=2 \mathrm{i} a b \mathrm{e}^{2 \mathrm{i} \omega t}+g_{1}^{(1)}(t), \\
& b:=\sqrt{\frac{a^{2}-\omega}{2}}
\end{aligned}
$$

where $g_{1}^{(1)}(t) \rightarrow 0$ as $t \rightarrow+\infty$, sufficiently fast. The intuition behind this assumption is the existence (for $b \in \mathbb{R})$ of the explicit solution of (2.1)

$$
q(x, t)=a \mathrm{e}^{2 \mathrm{i} b x+2 \mathrm{i} \omega t},
$$

for which $q(0, t)$ and $q_{x}(0, t)$ are indeed as in (3.1) and (3.2) with $g_{0}^{(1)} \equiv 0$ and $g_{1}^{(1)} \equiv 0$ (notice, however, that since $b$ is real, $q(x, t)$ in (3.3) does not decay as $x \rightarrow+\infty$ !).

Using this RHP, in $[4,5]$ the authors give the description of the long time behavior of $q(x, t)$, which appeared to be qualitatively different in three different sectors of the quarter plane $x>0, t>0$. The following theorem has been proved (here we formulate it in a slightly different way):

Theorem $1\left(\omega<-3 a^{2},[4,5]\right)$. Consider the Dirichlet initial boundary value problem for the focusing NLS equation in the quarter plane $x>0, t>0$ :

$$
\begin{array}{ll}
\mathrm{i} q_{t}+q_{x x}+2|q|^{2} q=0, & \\
q(x, 0)=q_{0}(x), & x \in[0, \infty), \\
q(0, t)=g_{0}(t), & t \in[0, \infty),
\end{array}
$$

where $q_{0}(x) \rightarrow 0$ as $x \rightarrow+\infty$ and $g_{0}(t)-a \mathrm{e}^{2 \mathrm{i} \omega t} \rightarrow 0$ as $t \rightarrow+\infty$ sufficiently fast, with $a>0$ and $\omega \in \mathbb{R}$ such that $\omega<-3 a^{2}$, and $q_{0}(0)=g_{0}(0)$. Assume that

(A) the solution $q(x, t)$ of (3.4) satisfies the following limiting condition:

$$
q_{x}(0, t)-2 \mathrm{i} a b \mathrm{e}^{2 \mathrm{i} \omega t} \rightarrow 0 \quad \text { as } t \rightarrow+\infty,
$$

where $b=\sqrt{\left(a^{2}-\omega\right) / 2}>0$,

(B) there are no solitons.

Then the quarter plane $x>0, t>0$ is divided into three sectors:

(i) $x>4 b t$,

(ii) $4(b-a \sqrt{2}) t<x<4 b t$, 
(iii) $0<x<4(b-a \sqrt{2}) t$,

where the asymptotics of $q(x, t)$ as $t \rightarrow+\infty$ has the following forms:

(i) For $x>4 b t$, the asymptotics has a quasi-linear dispersive character of ZakharovManakov type (decaying modulated oscillations):

$$
\begin{aligned}
q(x, t) \sim & t^{-1 / 2} \alpha\left(-\frac{x}{4 t}\right) \\
& \times \exp \left\{\frac{\mathrm{i} x^{2}}{4 t}+2 \mathrm{i} \alpha^{2}\left(-\frac{x}{4 t}\right) \log t+\mathrm{i} \phi\left(-\frac{x}{4 t}\right)\right\},
\end{aligned}
$$

where $\alpha(\xi)$ and $\phi(\xi)$ are determined in terms of the spectral functions associated with the initial values $q_{0}(x)$ and the boundary values $\left\{q(0, t), q_{x}(0, t)\right\}$.

(ii) For $4(b-a \sqrt{2}) t<x<4 b t$, the asymptotics has the form of a modulated elliptic wave expressed in terms of associated $\theta$-functions.

(iii) For $0<x<4(b-a \sqrt{2})$, the asymptotics has the form of a plane wave:

$$
q(x, t) \sim a \exp \left\{2 \mathrm{i}(b x+\omega t)-\phi\left(-\frac{x}{4 t}\right)\right\}
$$

with $\phi(0)=0$, where $\phi(\xi)$ is determined in terms of the spectral functions.

Remark 1. It is straightforward to incorporate solitons to this scheme.

3.2. Admissible data. The intuition behind the restriction $\omega<-3 a^{2}$ on the range of values of $\omega$ for which one expects the type of asymptotics described above, is the requirement that the plane wave region (iii) is not empty so that the $t$-axis lies in this region (on its boundary) and thus the assumed behavior of $q_{x}(0, t)$ is in agreement with the asymptotic result (3.7) (the limiting case $b=a \sqrt{2}$ corresponds to $\omega=-3 a^{2}$ ).

On the other hand, it is not a priori clear whether a particular value of the (complex) amplitude for $q_{x}(0, t)$ in (3.5) is only admissible or a different value $c \in \mathbb{C}$ may appear in the asymptotics for $q_{x}(0, t)$ :

$$
q_{x}(0, t)-c e^{2 \mathrm{i} \omega t} \rightarrow 0 \text { as } t \rightarrow \infty .
$$

In (3.5), the value of $c$ :

$$
c=2 \mathrm{i} a \sqrt{\frac{a^{2}-\omega}{2}}
$$

is chosen to be uniquely determined by $a$ and $\omega$.

Indeed, spectral functions can be associated to $g_{0}(t)$ and $g_{1}(t)$ satisfying respectively (3.1) and (3.8) with any $c \in \mathbb{C}$ independent of $a$ and $\omega$, in the framework of the scattering problem (on a periodic background) for equation $(2.4 \mathrm{~b})$. The main problem, however, lies in the compatibility of the set $\left\{q_{0}, g_{0}, g_{1}\right\}$.

It is natural to assume that the requirement that $\left\{q_{0}, g_{0}, g_{1}\right\}$ be compatible may affect the arbitrariness of $c$ in (3.8). Indeed, it can be shown [7] that the compatibility can be expressed, in the same way as in the case of decaying boundary conditions [16], in terms of a "global relation" amongst the associated spectral functions. Then the analysis of the global relation yields the following result:

Theorem 2 (admissible data, [7]). Let $q(x, t)$ be the solution of the IBV problem (3.4), where $g_{0}(t)-a \mathrm{e}^{2 \mathrm{i} \omega t} \rightarrow 0$ as $t \rightarrow \infty$ sufficiently fast, with $a>0$ and $\omega \in \mathbb{R}$. Assume that 
(A) $q_{x}(0, t)-c \mathrm{e}^{2 \mathrm{i} \omega t} \rightarrow 0$ as $t \rightarrow \infty$, where $c \in \mathbb{C}$.

Then the admissible values of $(a, \omega, c)$ are as follows:

- $\omega \in\left(-\infty,-3 a^{2}\right] \cup\left[\frac{a^{2}}{2}, \infty\right)$.

- The admissible values of $c$ are functions of $\omega$ and $a$ :

- For $\omega \leq-3 a^{2}, c=2 \mathrm{i} a \sqrt{\frac{a^{2}-\omega}{2}}$.

- For $\omega \geq \frac{a^{2}}{2}, c= \pm a \sqrt{2 \omega-a^{2}}$.

The proof of Theorem 2 is based on the analysis of the way how the global relation imposes additional analytical restrictions for the spectral functions associated with $\left\{g_{0}, g_{1}\right\}$ thus restricting the admissible behavior of $g_{1}(t)$ for large $t$.

3.3. Explicit solutions. An interesting observation is that the whole range of admissible cases for $\omega \geq \frac{a^{2}}{2}$ is illustrated by a family of explicit solutions related to "stationary solitons", which are solutions of initial value problems with decaying initial conditions as $|x| \rightarrow \infty$.

Indeed, apart from (3.3), the NLS equation has another family of explicit solutions whose values at $x=0$ are periodic exponentials of type (3.1). This family (parameterized by $\omega>0$ and $\left.\varphi_{0} \in \mathbb{R}\right)$ consists of stationary solitons:

$$
q(x, t) \equiv q\left(x, t ; \omega, \varphi_{0}\right)=\frac{\sqrt{2 \omega}}{\cosh \left(\sqrt{2 \omega} x+\varphi_{0}\right)} \mathrm{e}^{2 \mathrm{i} \omega t} .
$$

For these solutions we have

$$
q(0, t)=\frac{\sqrt{2 \omega}}{\cosh \varphi_{0}} \mathrm{e}^{2 \mathrm{i} \omega t} \equiv a \mathrm{e}^{2 \mathrm{i} \omega t}
$$

i.e., $q(0, t)$ has the form of (3.1). But now $a$ and $\omega$ are related by

$$
a=\frac{\sqrt{2 \omega}}{\cosh \varphi_{0}}
$$

and thus $a^{2} / 2 \leq \omega$. Notice that, in contrast with (3.3), $q(x, t)$ in (3.9) decays to 0 as $x \rightarrow+\infty$.

The values of $q_{x}(x, t)$ for $x=0$ have the form

$$
q_{x}(0, t)=\frac{-2 \omega \sinh \varphi_{0}}{\cosh ^{2} \varphi_{0}} \mathrm{e}^{2 \mathrm{i} \omega t}
$$

so that in this case we have

$$
q_{x}(0, t)=2 a \hat{b} \mathrm{e}^{2 \mathrm{i} \omega t}
$$

where

$$
\hat{b}:=-\sqrt{\frac{\omega}{2}} \tanh \varphi_{0}
$$

In terms of $\omega$ and $a$,

$$
4 \hat{b}^{2}=2 \omega-a^{2}
$$

and thus

$$
q_{x}(0, t)= \pm a \sqrt{2 \omega-a^{2}} \mathrm{e}^{2 \mathrm{i} \omega t},
$$

the sign \pm being opposite to the sign of $\varphi_{0}$.

Notice that in this case we have the possibility to assume two different values of $\hat{b}$ (with different sign), if $\hat{b} \neq 0$. A simple observation is that for $\varphi_{0}=0$ (i.e., for $\hat{b}=0$ ) we have 
$q_{x}(0, t) \equiv 0$ for a one-parameter family of solutions $q(x, t)$ of the NLS (parameterized by $\omega$ ), with $q(0, t)=\sqrt{2 \omega} \mathrm{e}^{2 \mathrm{i} \omega t}$. Therefore, for the IBV problem with Neumann boundary conditions at $x=0$ (which is also well-posed)

$$
\begin{array}{ll}
\mathrm{i} q_{t}+q_{x x}+2|q|^{2} q=0, & \\
q(x, 0)=q_{0}(x), & x \in[0, \infty), \\
q_{x}(0, t)=0, & t \in[0, \infty)
\end{array}
$$

we see that the possible behavior of $q(0, t)$ as $t \rightarrow+\infty$ is at least a one-parameter family $q(0, t) \sim \sqrt{2 \omega} \mathrm{e}^{2 \mathrm{i} \omega t}$ with $\omega>0$.

3.4. $\boldsymbol{\omega} \geq \boldsymbol{a}^{\mathbf{2}} / \mathbf{2}$. Coming back to the IBV problem with the Dirichlet boundary conditions, we have the following result.

Theorem $3\left(\omega \geq a^{2} / 2,[7]\right)$. Consider the Dirichlet initial boundary value problem (3.4) for the focusing NLS equation in the quarter plane $x>0, t>0$, where $q_{0}(x) \rightarrow 0$ as $x \rightarrow \infty$ and $g_{0}(t)-a \mathrm{e}^{2 \mathrm{i} \omega t} \rightarrow 0$ as $t \rightarrow+\infty$ sufficiently fast, with $a>0$ and $\omega \in \mathbb{R}$ such that $\omega \geq a^{2} / 2$, and $q_{0}(0)=g_{0}(0)$. Assume that

(A) the solution $q(x, t)$ of (3.4) satisfies the limiting condition (3.8), with $c=a \sqrt{2 \omega-a^{2}}$ or with $c=-a \sqrt{2 \omega-a^{2}}$,

(B) there are no solitons propagating in the quarter plane $x>0, t>0$.

Then for any $\varepsilon>0$, the asymptotics of $q(x, t)$ as $t \rightarrow+\infty$ in the region $x / t>\varepsilon$ has a quasilinear dispersive character of Zakharov-Manakov type ("decaying modulated oscillations") (3.6), where $\alpha(\xi)$ and $\varphi(\xi)$ are determined in terms of spectral functions associated with the initial values $q_{0}(x)$ and the boundary values $\left\{q(0, t), q_{x}(0, t)\right\}$.

Remark 2. In spectral terms, solitons propagating in the quarter plane (i.e., showing themselves along certain lines $x / t=\varphi_{i}$ in the $(x, t)$ quarter plane) correspond to zeros, with nonzero real parts, of the spectral functions associated with the initial and boundary values. Similarly to the case of Theorem 1, one can incorporate such solitons into the asymptotics applying the dressing method [18].

Although the results of Theorems 1-3 correspond to a conditional setting (we are given the Dirichlet values of $q(x, t), q_{0}(x)$ and $g_{0}(t)$, and we make assumption (3.8) about the class of $\left.q_{x}(0, t)\right)$, the results of the numerical simulations, where the Dirichlet initial boundary value problem (3.4) is solved for $q(x, t)$, show very good agreement between the values of $q_{x}(0, t)$ calculated from $q(x, t)$ and the values of admissible asymptotics in Theorem 2, see Section 4 .

3.5. $-\mathbf{3 a}^{\mathbf{2}}<\boldsymbol{\omega}<\boldsymbol{a}^{\mathbf{2}} / \mathbf{2}$. As for the parameter range $-3 a^{2}<\omega<\frac{a^{2}}{2}$, we believe that theoretical reasons for the form of the asymptotics of $q(0, t)$ and $q_{x}(0, t)$ compatible with the fact that $q(0, t)$ and $q_{x}(0, t)$ are indeed boundary values of a solution $q(x, t)$ of the NLS equation, lie in the following circle of ideas.

3.5.1. Related initial value problems on the line. Consider the initial value problem (on the whole line) for the NLS equation with initial data $q_{0}(x)$ having (generally) different behaviors when $x \rightarrow+\infty$ and when $x \rightarrow-\infty$.

More precisely, assume that $q_{0}(x) \rightarrow 0$ when $x \rightarrow+\infty$ while $q_{0}(x)$ approaches a non-zero background, of a certain structure, when $x \rightarrow-\infty$. Assume that we are able to analyze the 
long time asymptotics of the solution $q(x, t)$ of this problem. Then the boundary values of this solution, i.e., $q(0, t)$ and $q_{x}(0, t)$, suggest admissible asymptotics for the boundary values $g_{0}(t)$ and $g_{1}(t)$ of our initial boundary value problem on the half-line, i.e., in the domain $x>0, t>0$.

3.5.2. Specific related initial value problem [8]. Particularly, for $q_{0}(x)$ such that:

- $q_{0}(x) \rightarrow 0$ as $x \rightarrow+\infty$,

- $q_{0}(x)-a \mathrm{e}^{2 \mathrm{i} b x} \rightarrow 0$ as $x \rightarrow-\infty$,

with $a>0$ and $b \in \mathbb{R}$, we are able [8] to study the long time asymptotics of the solution of the initial value problem (on the whole line $x \in(-\infty, \infty)$ ) for the NLS equation (2.1). The results are that there exist three regions (sectors) in the half-plane $x \in(-\infty, \infty), t>0$, where the solution behaves similarly to the way presented in Theorem 1 .

Namely, for $x<4(b-a \sqrt{2}) t$ we have

$$
q(x, t) \sim a \exp \left\{2 \mathrm{i}(b x+\omega t)-\phi\left(-\frac{x}{4 t}\right)\right\},
$$

where $\omega, a$, and $b$ are related as in Theorem 1:

$$
\omega=a^{2}-2 b^{2} .
$$

Hence, if $b>0$ and $b>a \sqrt{2}-$ thus $\omega<-3 a^{2}$ - then the plane wave sector overlaps the quarter plane $x>0, t>0$, and thus the main term of the asymptotics of the solution of the initial value problem as $t \rightarrow+\infty$ along the line $x=0$ has the form (3.7).

Otherwise, the asymptotics along the line $x=0$ is described in terms of $\theta$-functions corresponding to 1-gap algebro-geometric solutions.

3.5.3. Conjecture. This allows conjecturing the appearance of 1-gap (or, more generally, $n$ gap) formulas for the asymptotics of $q_{x}(0, t)$ and, consequently, for $q(x, t)$ in sectors of the quarter plane $x>0, t>0$, as solutions of the initial boundary value problem.

\section{NumERics}

In this section, we present four groups of results of numerical simulations illustrating the theoretical considerations and conjectures above. In all calculations - except for Examples 4, 8,9 - the initial boundary value problem (3.4) is solved numerically for the following initial and boundary conditions (if otherwise is not stated explicitly):

$$
\begin{aligned}
& q_{0}(x)=p \mathrm{e}^{-x^{2}}, \\
& g_{0}(t)=a \mathrm{e}^{2 \mathrm{i} \omega t}+\left[p-a-\mathrm{i}\left(2 p-2 p^{3}+2 a \omega\right) t\right] \mathrm{e}^{-10 t^{2}},
\end{aligned}
$$

in order to have good compatibility of initial and boundary values at $(0,0)$. The computational interval is set to $[0,200]$, terminated by zero Dirichlet boundary condition. The Schrödinger equation is evolved with the relaxation scheme proposed by Besse [1]. The spatial step size and the time step are set to 0.005 and 0.001 , respectively.

We consider four classes of numerical tests:

(i) The $1^{\text {st }}$ group illustrates the parameter range $\omega \leq-3 a^{2}$.

(ii) The $2^{\text {nd }}$ one is for the range $\omega \geq \frac{a^{2}}{2}$. 
(iii) The $3^{\text {rd }}$ group gives examples in the intermediate range $-3 a^{2}<\omega<\frac{a^{2}}{2}$, where the asymptotics of $q_{x}(0, t)$ cannot have the form $c \mathrm{e}^{2 \mathrm{i} \omega t}$.

(iv) The $4^{\text {th }}$ group corresponds to periodic boundary conditions that are different from the exponential form above.

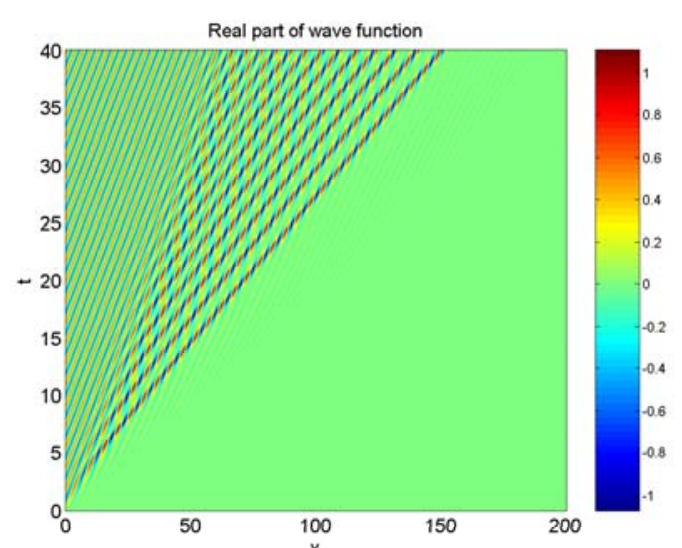

(a) values of $\operatorname{Re} q(x, t)$

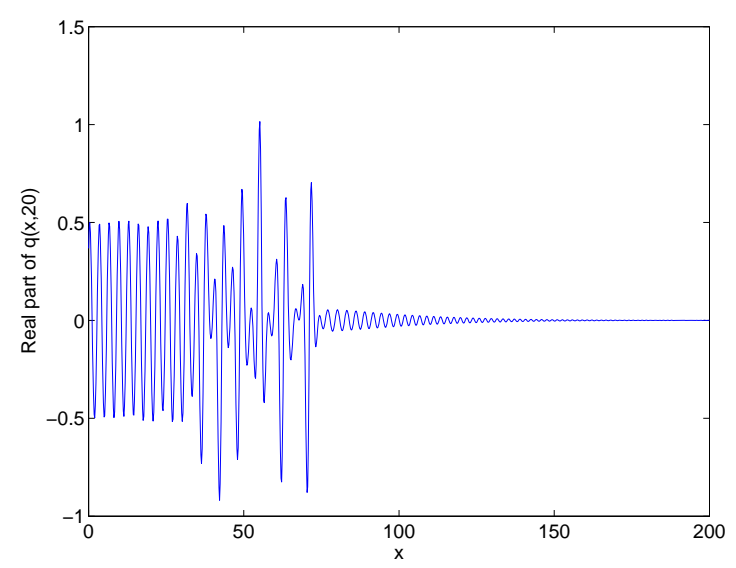

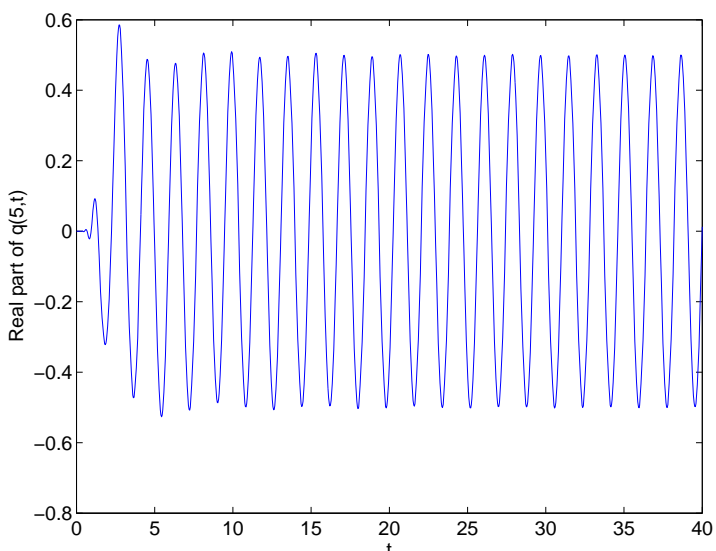

(b) values of $\operatorname{Re} q(5, t)$

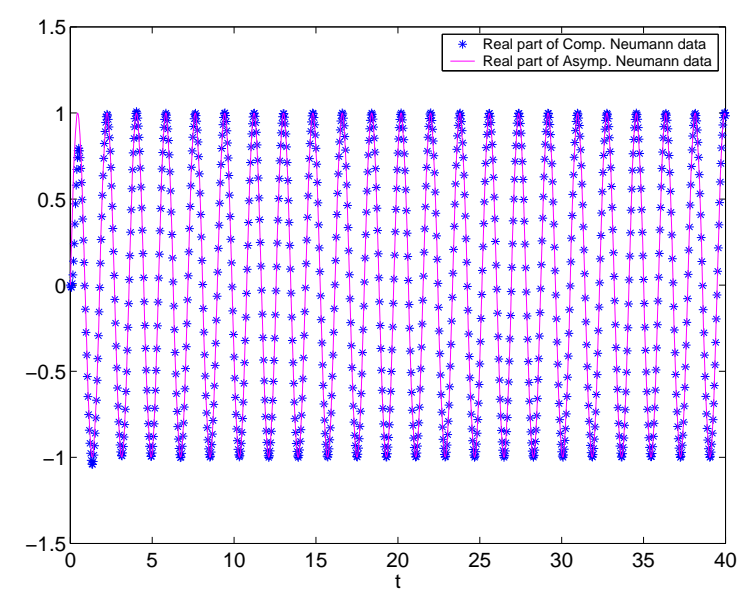

Figure 1. Example 1: $a=0.5, p=0, \omega=-1.75<-3 a^{2}$

4.1. Range $\boldsymbol{\omega} \leq-\mathbf{3} \boldsymbol{a}^{\mathbf{2}}$. This parameter range is illustrated by two numeric examples:

(i) Example 1, Figure 1 corresponds to $a=0.5, p=0$, and $\omega=-1.75$.

(ii) Example 2, Figure 2, corresponds to $a=0.5, p=1$, and $\omega=-1$.

All numeric examples in this range show:

- Three sectors in the $(x, t)$ plane with qualitatively different behavior (in accordance with Theorem 1), see Figures 1(a) and 2(a).

- Very good agreement of calculated $q_{x}(0, t)$ with (3.5), see Figures $1(\mathrm{~d})$ and $2(\mathrm{~d})$. 


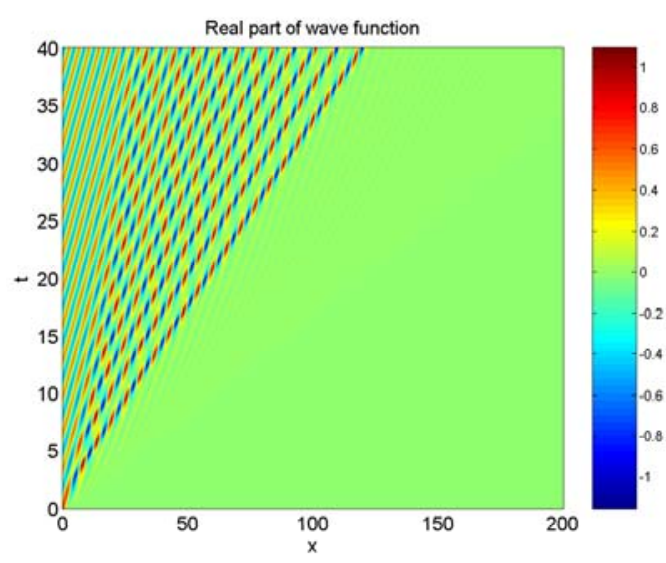

(a) values of $\operatorname{Re} q(x, t)$

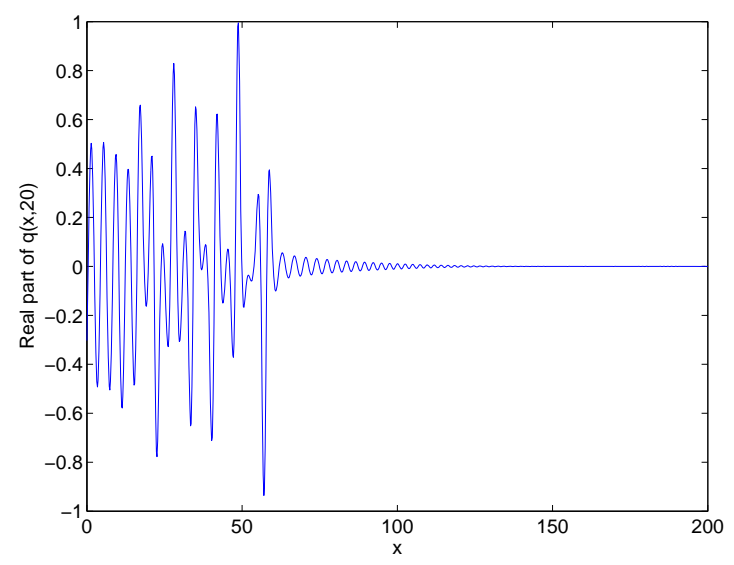

(c) values of $\operatorname{Re} q(x, 20)$

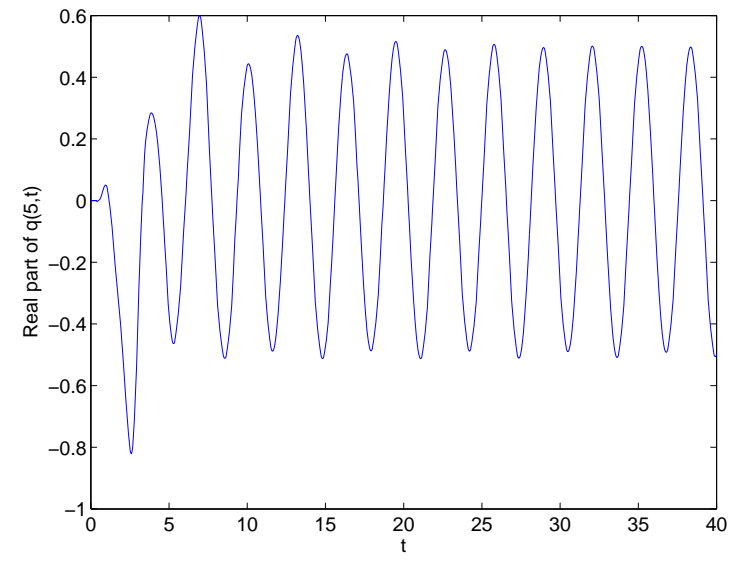

(b) values of $\operatorname{Re} q(5, t)$

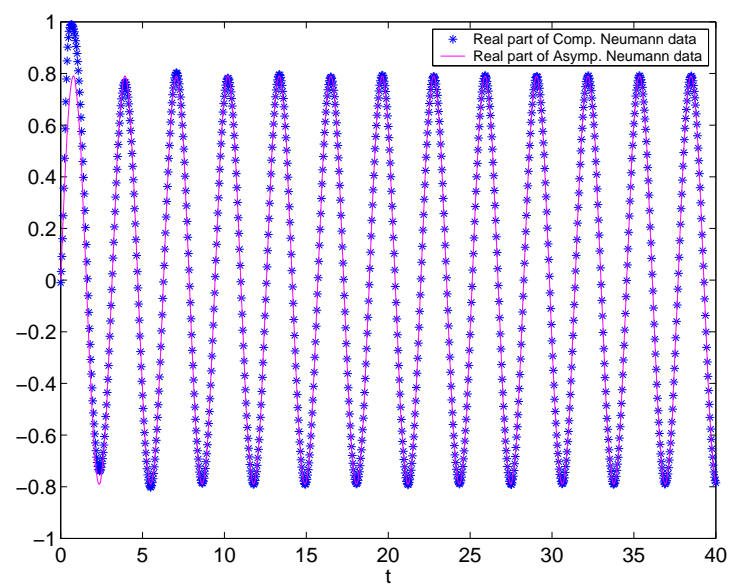

(d) $\operatorname{Re} q_{x}(0, t)$ against asymptotics $(3.5)$

Figure 2. Example 2: $a=0.5, p=1, \omega=-1<-3 a^{2}$

- Rapid decay as $x$ is growing, with fixed $t$, see Figures $1(\mathrm{c})$ and 2 (c)).

- Sinusoidal character in the plane wave sector: for fixed $x$, see Figures 1 (b) and 2(b).

Numerical experiments in the range $\omega \leq-3 a^{2}$ show that, although the asymptotics of the Neumann values (3.5) is justified theoretically only assuming that it has a one-frequency character (see Theorem 2), it indeed takes place in a wide set of initial and boundary conditions for the Dirichlet problem (the question how general is this set remains open).

4.2. Range $\omega \geq \frac{a^{2}}{2}$. This range is also illustrated by two numeric examples:

(iii) Example 3, Figure 3 corresponds to $a=0.5, p=1$, and $\omega=1$.

(iv) Example 4, Figure 4 corresponds to a stationary soliton (3.9) with $\omega=0.5$ and $\varphi_{0}=-1$, i.e., with $a=(\cosh 1)^{-1} \approx 0.648$.

Figures 3 shows: 


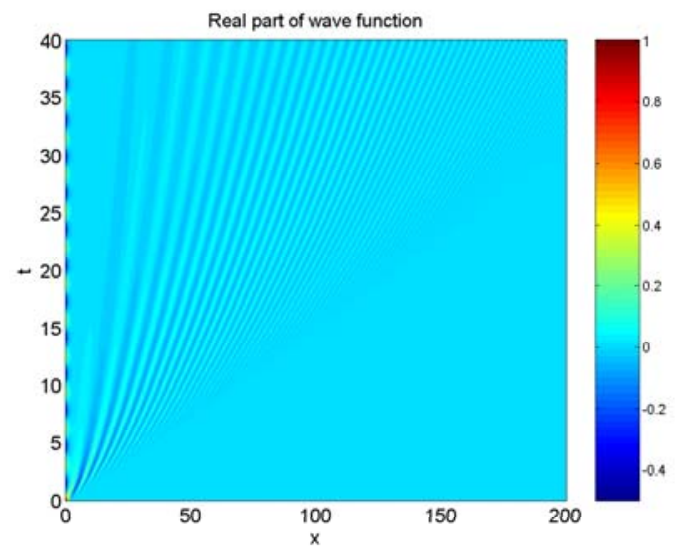

(a) values of $\operatorname{Re} q(x, t)$

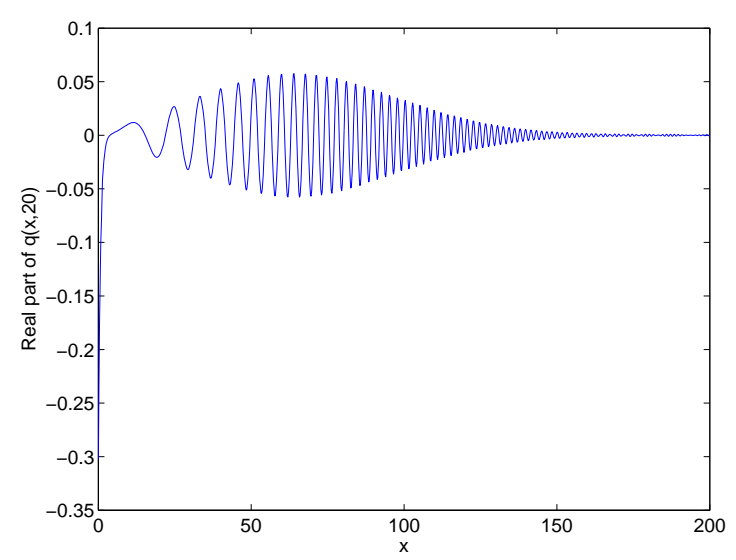

(c) values of $\operatorname{Re} q(x, 20)$

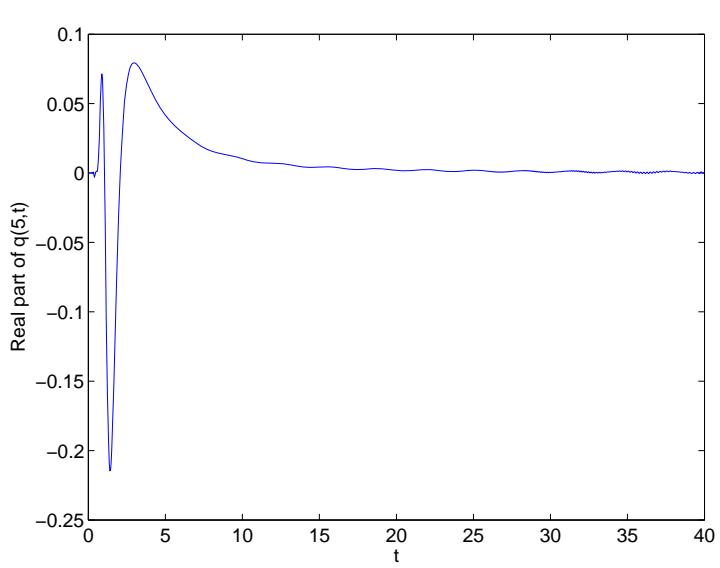

(b) values of $\operatorname{Re} q(5, t)$

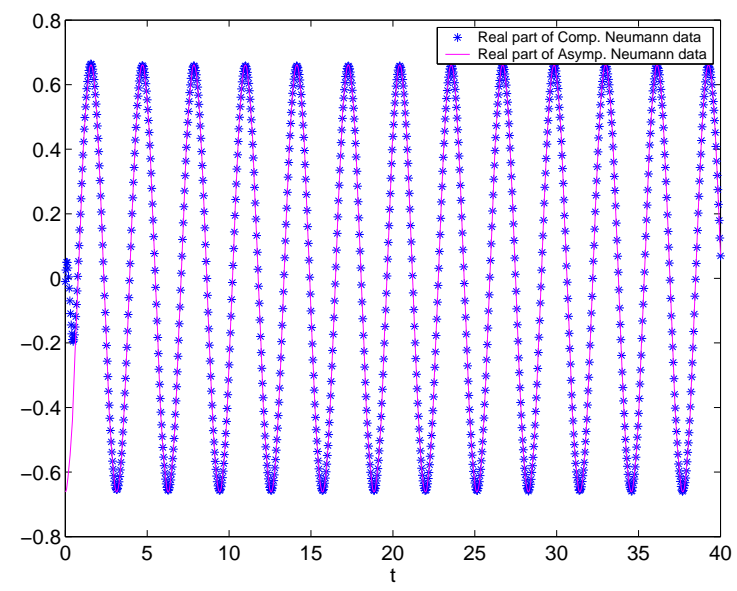

(d) $\operatorname{Re} q_{x}(0, t)$ against the asymptotics (3.8) with $c=-a \sqrt{2 \omega-a^{2}}$

Figure 3. Example 3: $a=0.5, p=1, \omega=1>a^{2} / 2$

- Rapid decay of $q(x, t)$ in the whole quadrant $x>0, t>0$ (away from the $t$-axis), see Figures 3(a), 3(b), and 3(c), which is in accordance with Theorem 3 (the oscillating pattern in Figure 3(c) is due to smaller terms of the asymptotics).

- Very good agreement of $q_{x}(0, t)$ with $(3.8)$, for $c=-a \sqrt{2 \omega-a^{2}}$, see Figure 3(d).

On the other hand, Figure 4 shows that the case $c=a \sqrt{2 \omega-a^{2}}$, though admissible theoretically (explicit examples for this case are given by (3.9) with $\varphi_{0}<0$ ), is unstable. Indeed, Figure 4 illustrates the values of $q_{x}(0, t)$, where $q(x, t)$ is the solution of the Dirichlet problem (3.4) with $q_{0}(x)$ and $g_{0}(t)$ being exactly as coming from (3.9) with $\omega=0.5$ and $\varphi_{0}=-1$ (which corresponds to $\left.a=(\cosh 1)^{-1} \approx 0.648\right)$. It is seen that the calculated values of $q_{x}(0, t)$, being in good agreement for small $t$ with its true values $a \sqrt{2 \omega-a^{2}} \mathrm{e}^{2 \mathrm{i} \omega t}$, switch, for larger $t$, to $q_{x}(0, t) \approx-a \sqrt{2 \omega-a^{2}} \mathrm{e}^{2 \mathrm{i} \omega t}$. 


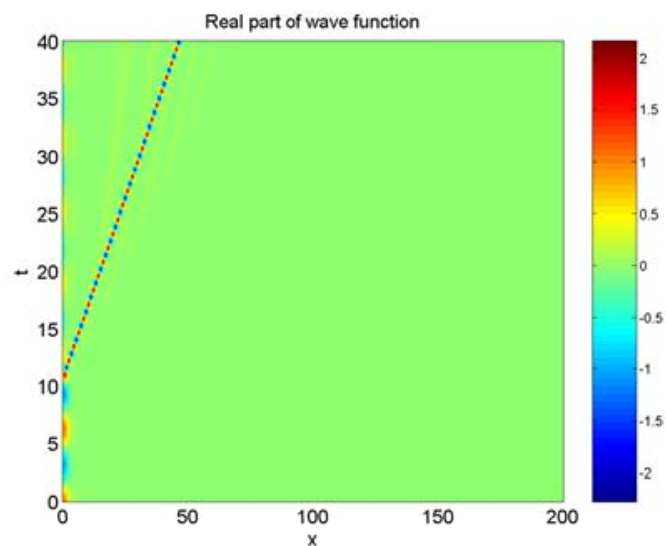

(a) values of $\operatorname{Re} q(x, t)$

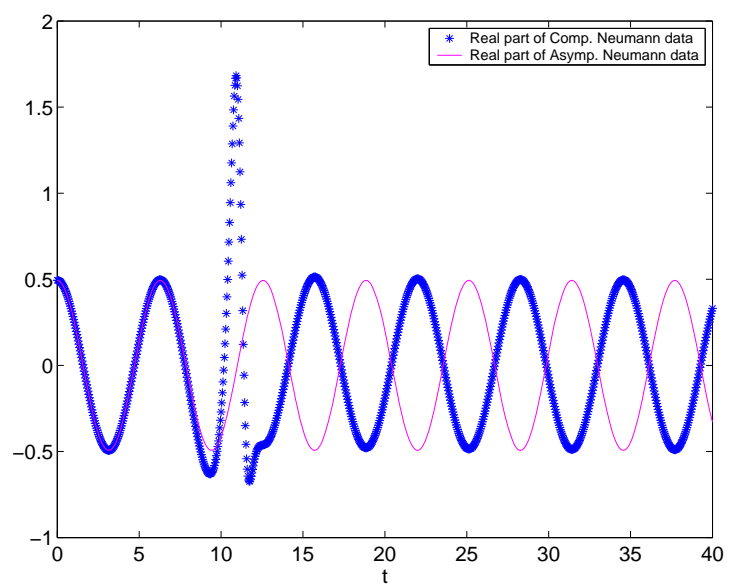

(c) values of $\operatorname{Re} q_{x}(0, t)$

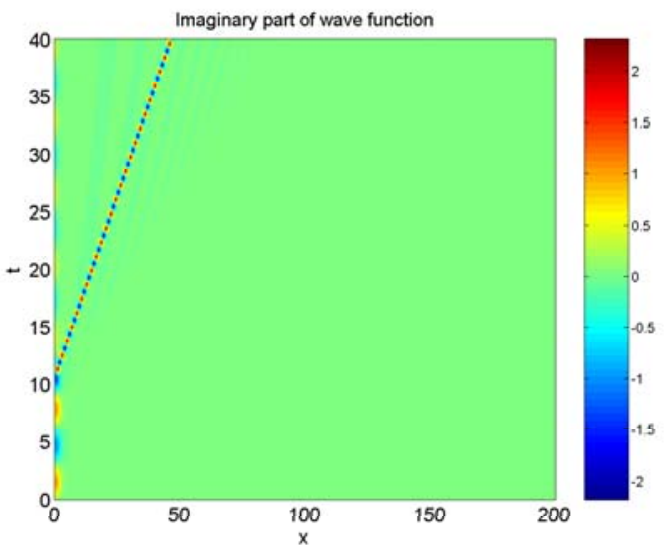

(b) values of $\operatorname{Im} q(x, t)$

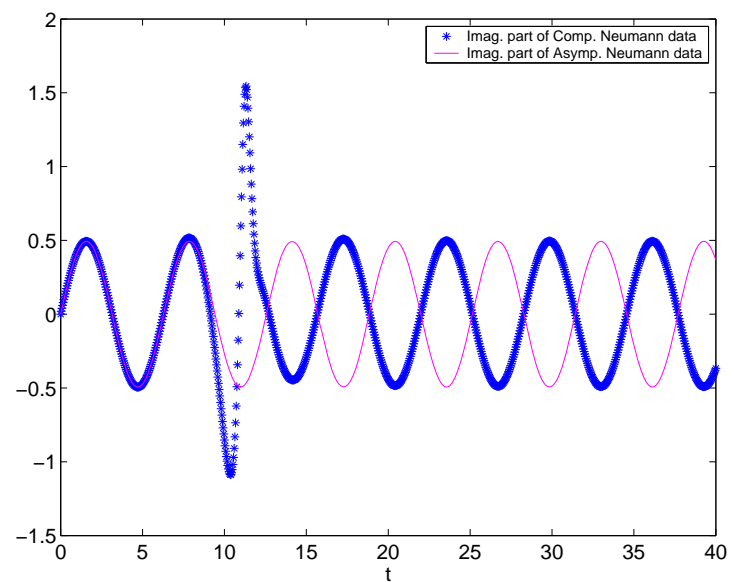

(d) values of $\operatorname{Im} q_{x}(0, t)$

Figure 4. Example 4: Instability of $q_{x}(0, t)=a \sqrt{2 \omega-a^{2}} \mathrm{e}^{2 \mathrm{i} \omega t}$

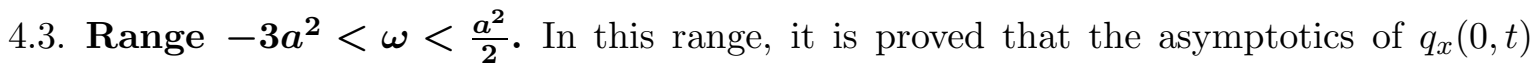
cannot have the one-frequency form (3.8).

4.3.1. $\omega=0$. Of particular interest is the case $\omega=0$, where the Dirichlet boundary data reduce (asymptotically) to a constant:

$$
q(0, t)-a \rightarrow 0 .
$$

Figures 5-6 illustrate this case for two different values of $a$ :

(v) Example 5, Figure 5 corresponds to $a=0.5, \omega=0, p=0$.

(vi) Example 6, Figure 6 corresponds to $a=1, \omega=0, p=0$.

It is seen that:

- The Neumann values $q_{x}(0, t)$ are (asymptotically) periodic, but not sinusoidal.

- There are two sectors in the $(x, t)$ plane: 


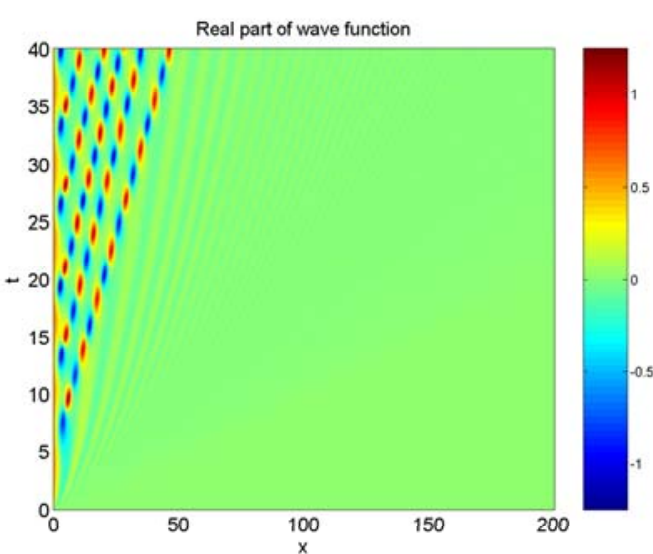

(a) values of $\operatorname{Re} q(x, t)$

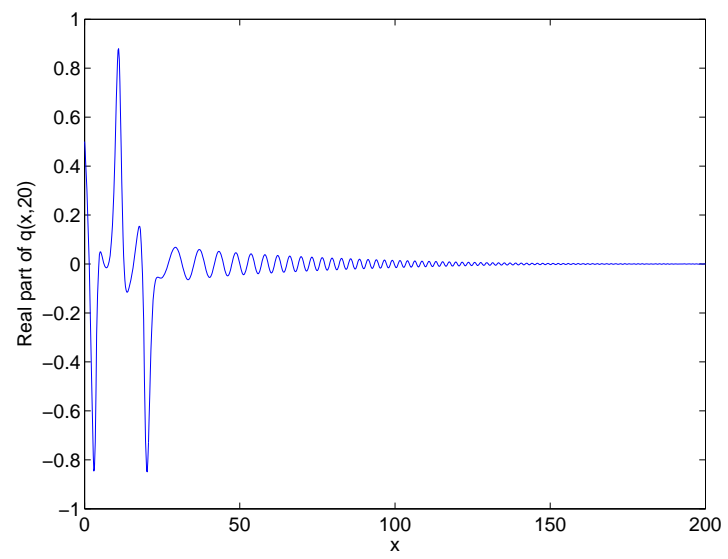

(c) values of $\operatorname{Re} q(x, 20)$

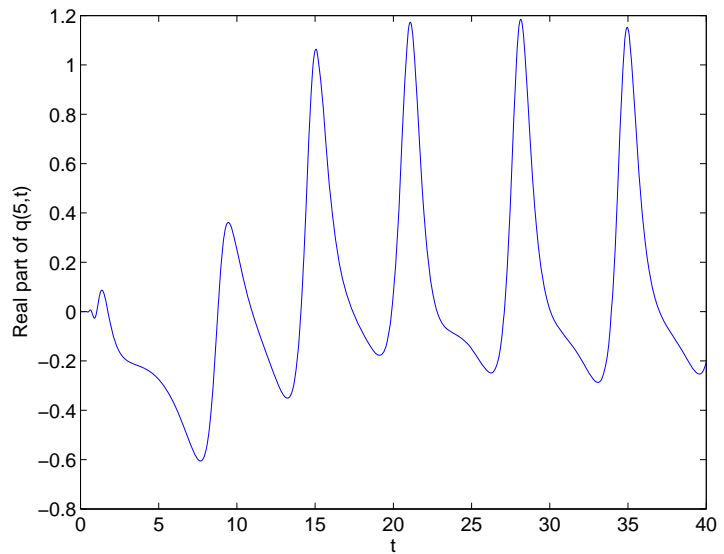

(b) values of $\operatorname{Re} q(5, t)$

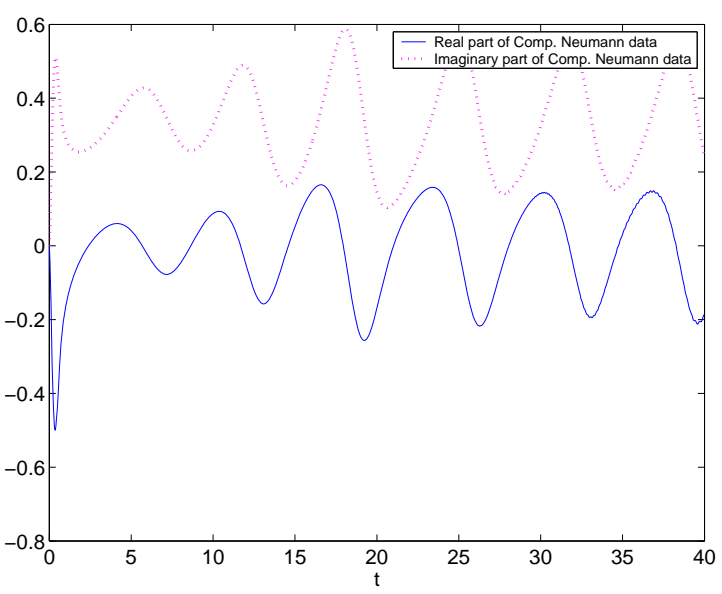

(d) values of $\operatorname{Re} q_{x}(0, t)$ and $\operatorname{Im} q_{x}(0, t)$

Figure 5. Example 5: $a=0.5, p=0, \omega=0$

(i) a sector of rapid decay,

(ii) a sector of oscillations similar to those in the elliptic wave zone in the case $\omega<-3 a^{2}$.

- For bigger $a$, see Example 6, Figure 6, the periodic character of $q_{x}(0, t)$ as well as of $q(x, t)$ for a fixed $x$ becomes more distinct.

4.3.2. $\omega \neq 0$. Figures 7 illustrate this case for one value of $a$ :

(vii) Example 7, Figure 7 corresponds to $a=0.5, p=0$, and $\omega=-0.5$.

Figure 7 shows that the picture for $\omega \neq 0$ in the considered range (particularly, for $\omega=-0.5$ and $a=0.5)$ is quite similar to that for $\omega=0$ :

- The Neumann values $q_{x}(0, t)$ are (asymptotically) periodic, but not sinusoidal.

- There are two sectors in the $(x, t)$ plane:

(i) a sector of rapid decay, 


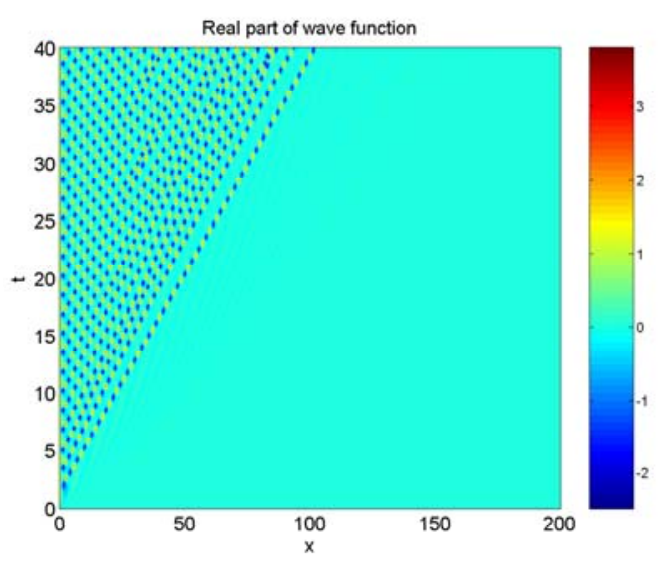

(a) values of $\operatorname{Re} q(x, t)$

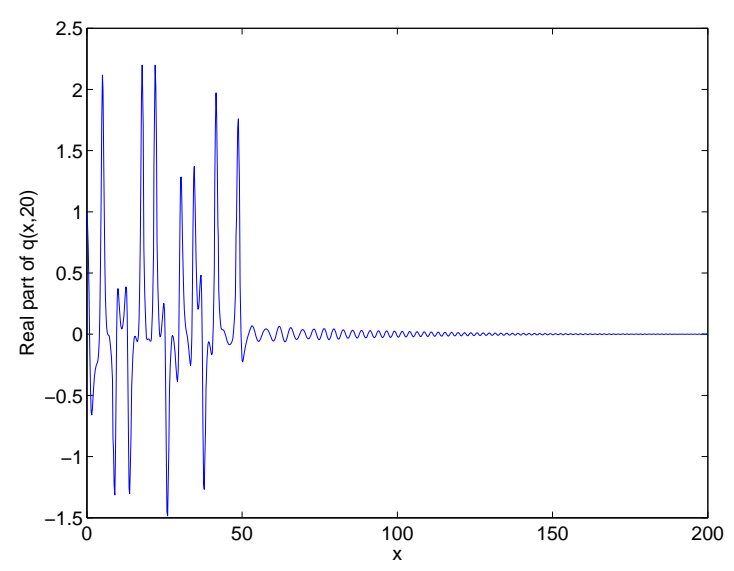

(c) values of $\operatorname{Re} q(x, 20)$

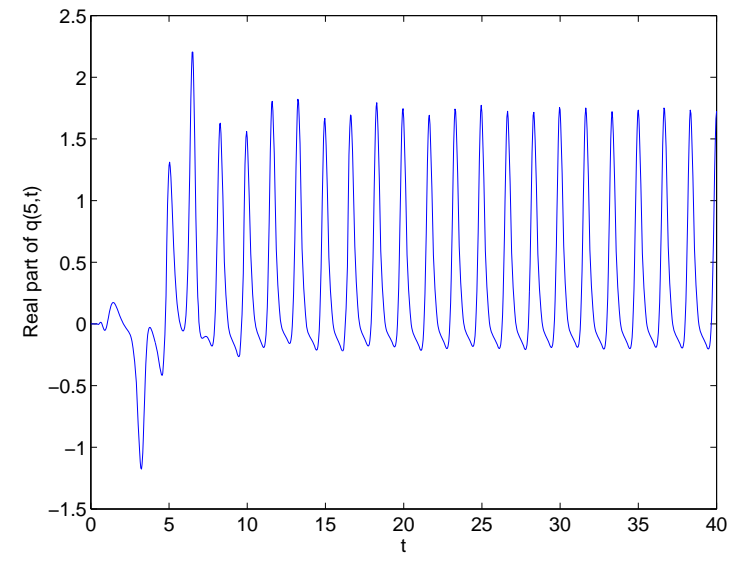

(b) values of $\operatorname{Re} q(5, t)$

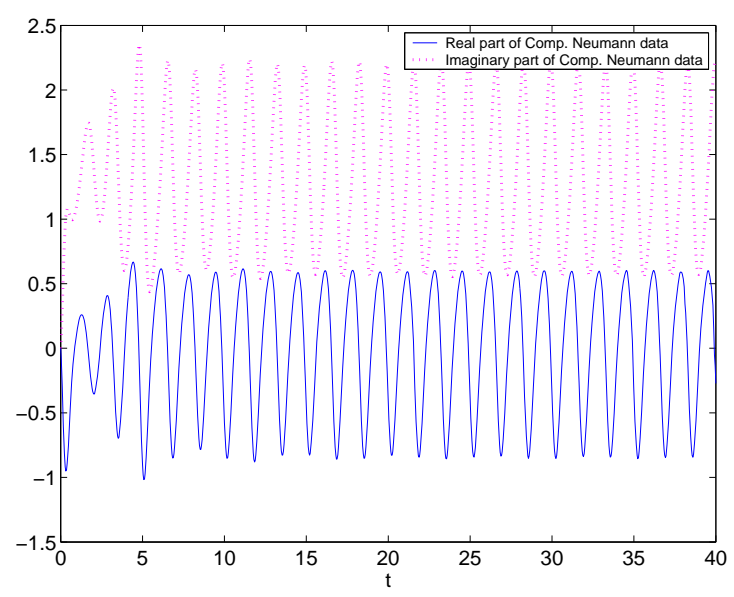

(d) values of $\operatorname{Re} q_{x}(0, t)$ and $\operatorname{Im} q_{x}(0, t)$

Figure 6. Example 6: $a=1, p=0$, and $\omega=0$

(ii) a sector of oscillations.

4.4. Boundary conditions of different type. For Figures 8-9, the boundary data were taken to be (asymptotically) different from $a \mathrm{e}^{2 \mathrm{i} \omega t}$ but having a similar (one-frequency) character:

$$
g_{0}(t)=\operatorname{Im}\left(a \mathrm{e}^{2 \mathrm{i} \omega t}-a \mathrm{e}^{-10 t^{2}}-2 \mathrm{i} a \omega t \mathrm{e}^{-10 t^{2}}\right) .
$$

(viii) Example 8 has the same parameters for the boundary values as in Example 1, i.e., $a=0.5$ and $\omega=-1.75$, see Figure 8.

(ix) Example 9 has the same parameters for the boundary values as in Example 3, i.e., $a=0.5$ and $\omega=1$, see Figure 9.

For both examples the initial conditions are the same: $q_{0}(x) \equiv 0$. 


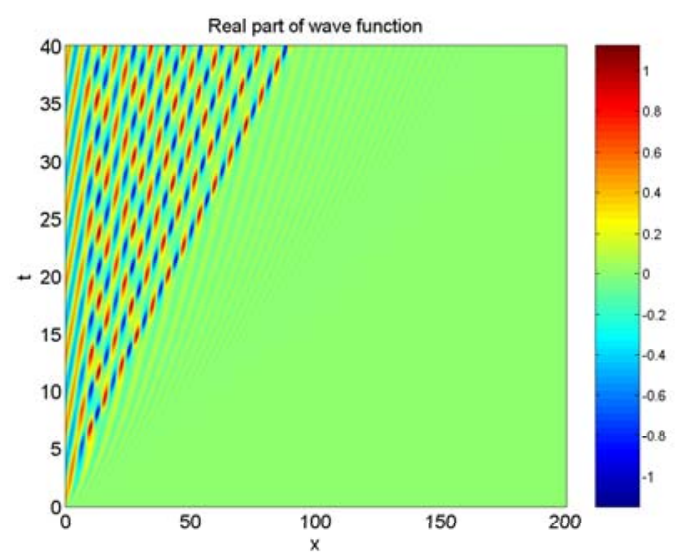

(a) values of $\operatorname{Re} q(x, t)$

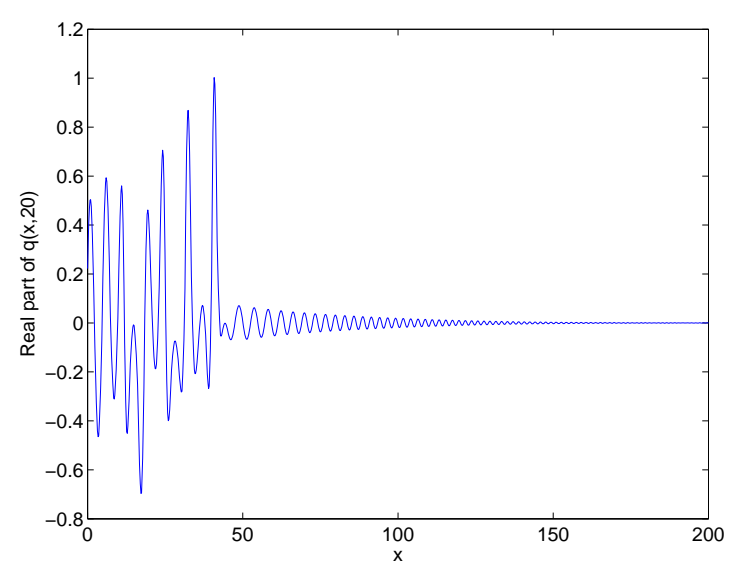

(c) values of $\operatorname{Re} q(x, 20)$

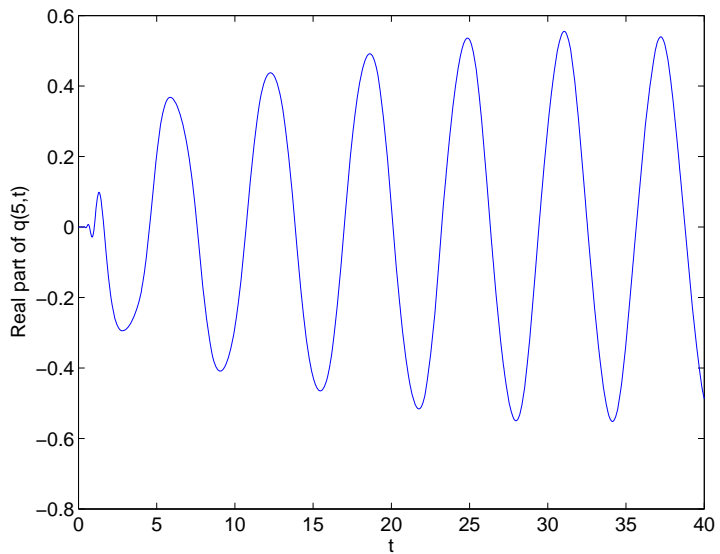

(b) values of $\operatorname{Re} q(5, t)$

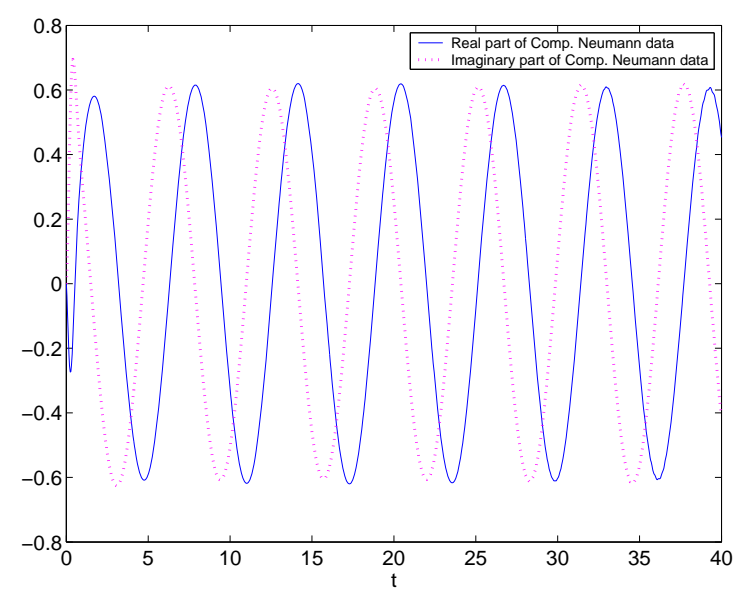

(d) values of $\operatorname{Re} q_{x}(0, t)$ and $\operatorname{Im} q_{x}(0, t)$

Figure 7. Example 7: $a=0.5, p=0, \omega=-0.5$

The pictures have similar features (which is in sharp contrast with the case of the boundary data of the form $a \mathrm{e}^{2 \mathrm{i} \omega t}$, cf. Figures $1(\mathrm{a}), 1(\mathrm{~d}), 3(\mathrm{a})$, and 3(d), which in turn are similar to those in the case of $q(0, t) \simeq a \mathrm{e}^{2 \mathrm{i} \omega t}$ with $\omega<-3 a^{2}$ : the three sectors are clearly present.

\section{CONCLUDING REMARKS}

For the two ranges $\omega \leq-3 a^{2}$ and $\omega \geq \frac{a^{2}}{2}$ our numerical experiments match perfectly with our theoretical results and with our assumptions on the Dirichlet-to-Neumann map.

For the intermediate range $-3 a^{2}<\omega<\frac{a^{2}}{2}$ our numerical experiments suggest asymptotics results like in the range $\omega \leq-3 a^{2}$. But the Dirichlet-to-Neumann map is clearly of a different type than in the two previous cases and the numerics do not suggest any simple formula for the Neumann data. We can only predict the appearance of $n$-gap formulas for the asymptotics of $q_{x}(0, t)$. 


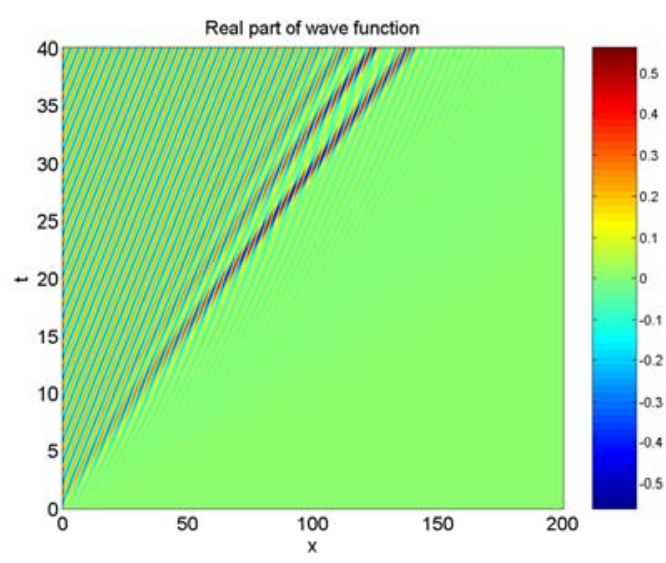

(a) values of $\operatorname{Re} q(x, t)$

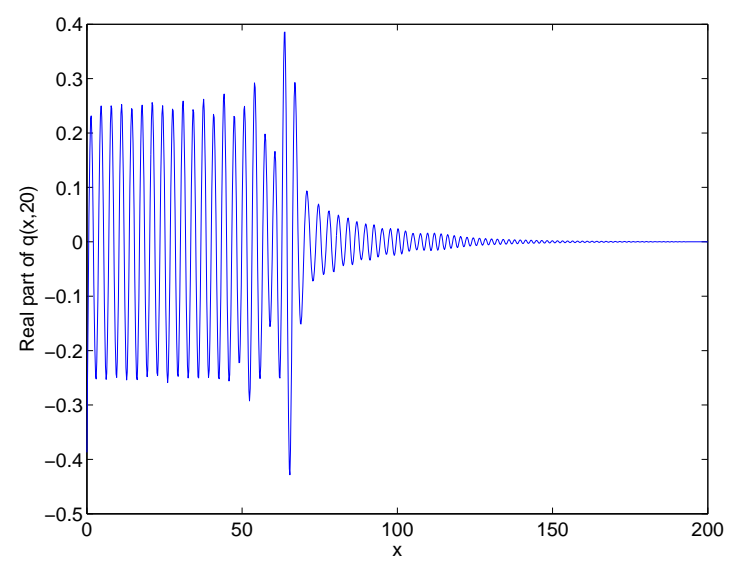

(c) values of $\operatorname{Re} q(x, 20)$

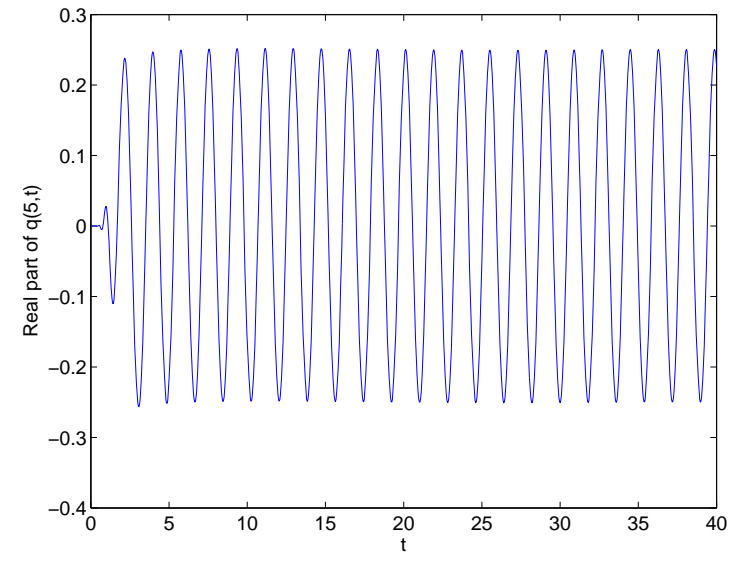

(b) values of $\operatorname{Re} q(5, t)$

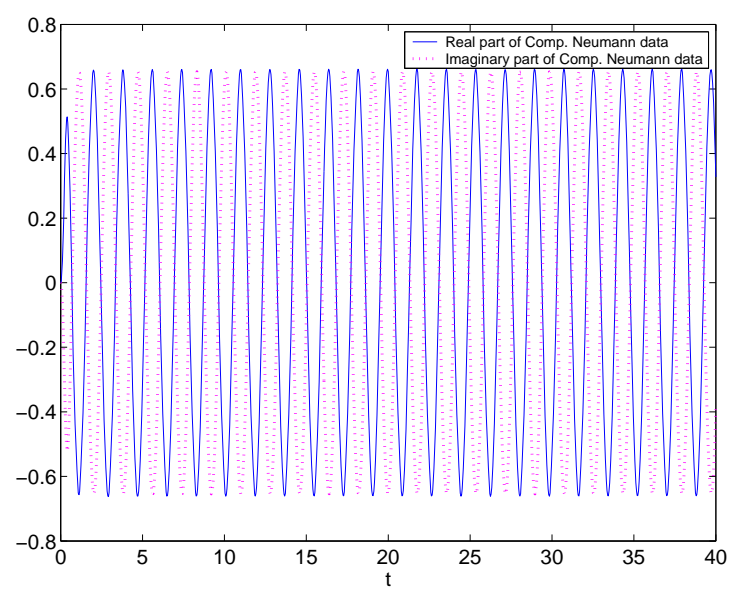

(d) values of $\operatorname{Re} q_{x}(0, t)$ and $\operatorname{Im} q_{x}(0, t)$

Figure 8. Example 8: $q(0, t) \simeq a \sin 2 \omega t$ with $a=0.5$ and $\omega=-1.75$

\section{ACKNOWLEDGEMENTS}

The work of Chunxiong Zheng was partially supported by the National Natural Science Foundation of China under Grant No. 10401020.

\section{REFERENCES}

[1] C. Besse, A relaxation scheme for the nonlinear Schrödinger equation, SIAM J. Numer. Anal. 42 (2004), no. 3, 934-952.

[2] A. Boutet de Monvel, A. S. Fokas, and D. Shepelsky, Analysis of the global relation for the nonlinear Schrödinger equation on the half-line, Lett. Math. Phys. 65 (2003), no. 3, 199-212.

[3] - The mKdV equation on the half-line, J. Inst. Math. Jussieu 3 (2004), no. 2, 139-164.

[4] A. Boutet de Monvel, A. R. Its, and V. Kotlyarov, Long-time asymptotics for the focusing NLS equation with time-periodic boundary condition, C. R. Math. Acad. Sci. Paris 345 (2007), no. 11, 615-620. 


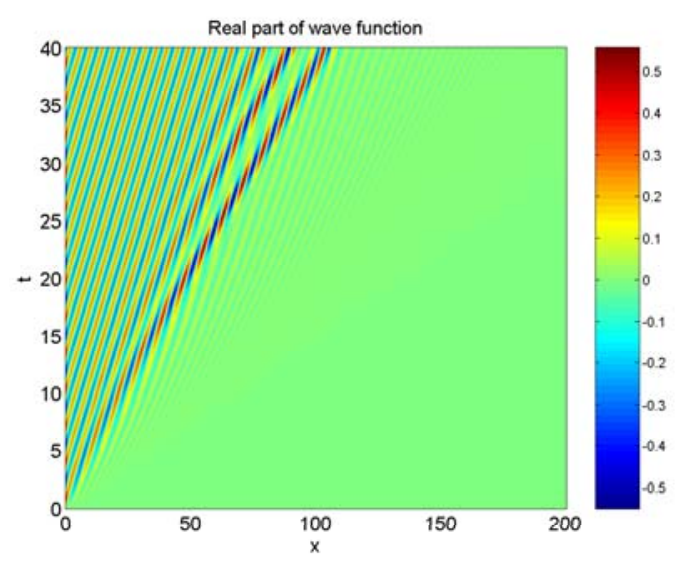

(a) values of $\operatorname{Re} q(x, t)$

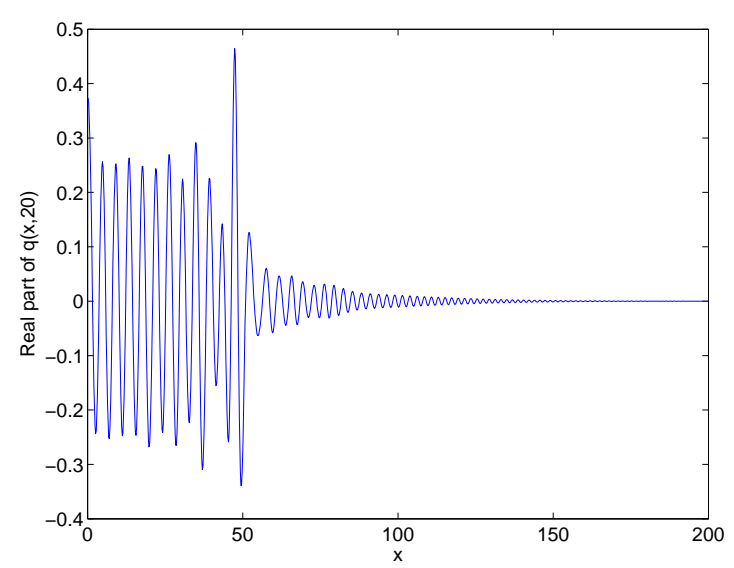

(c) values of $\operatorname{Re} q(x, 20)$

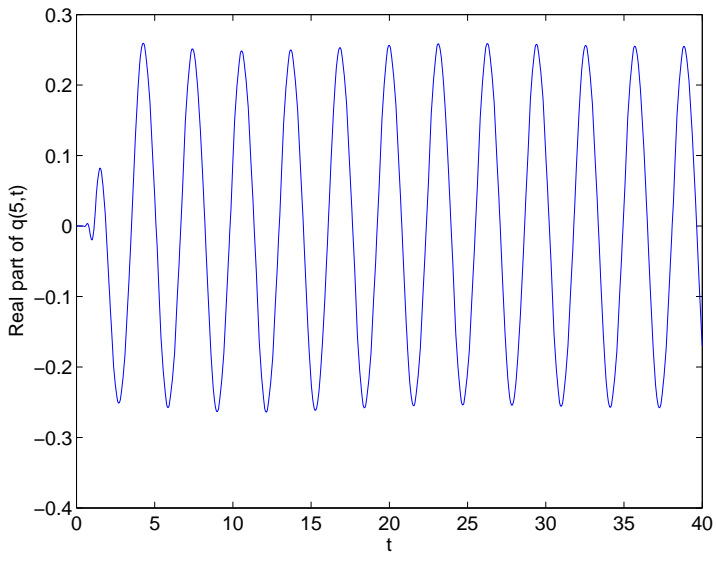

(b) values of $\operatorname{Re} q(5, t)$

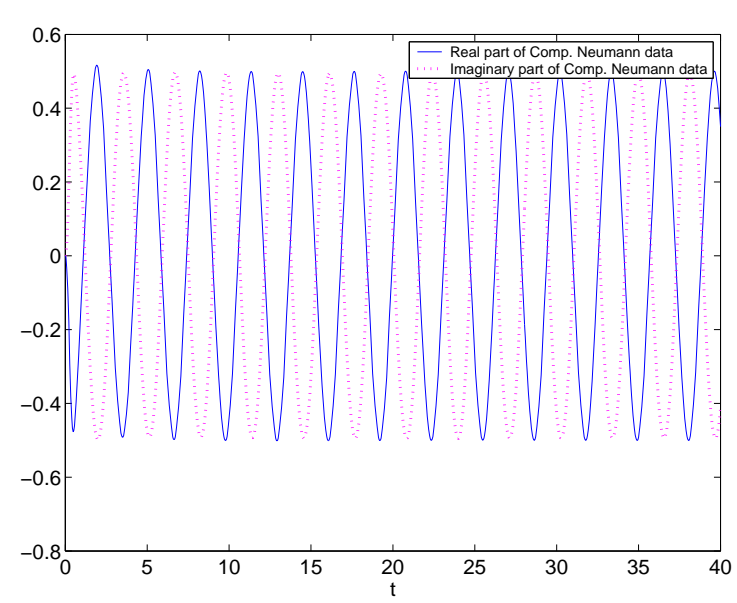

(d) values of $\operatorname{Re} q_{x}(0, t)$ and $\operatorname{Im} q_{x}(0, t)$

Figure 9. Example 9: $q(0, t) \simeq a \sin 2 \omega t$ with $a=0.5$ and $\omega=1$

[5] L Long-time asymptotics for the focusing NLS equation with time-periodic boundary condition on the half-line (2008), preprint $\mathrm{BiBoS} \mathrm{n}^{\circ} 08-04-287$.

[6] A. Boutet de Monvel and V. Kotlyarov, The Focusing Nonlinear Schrödinger Equation on the Quarter Plane with Time-Periodic Boundary Condition: a Riemann-Hilbert approach, J. Inst. Math. Jussieu 6 (2007), no. 4, 579-611.

[7] A. Boutet de Monvel, V. P. Kotlyarov, and D. Shepelsky, The focusing NLS equation with periodic Dirichlet data: admissible Neumann values and long-time asymptotics (2008), preprint BiBoS.

[8] - Focusing NLS equation: long time evolution of step-like initial data (2008), preliminary version.

[9] A. Boutet de Monvel and D. Shepelsky, Initial boundary value problem for the mKdV equation on a finite interval, Ann. Inst. Fourier (Grenoble) 54 (2004), no. 5, 1477-1495, xv, xxi.

[10] L L L L L L - L time asymptotics of the Camassa-Holm equation on the line, Proceedings of the Conference on Integrable Systems, Random Matrices, and Applications: A conference in honor of Percy Deift's 60th birthday (New York, NY, 2007), Contemp. Math., vol. 458, Amer. Math. Soc., Providence, RI, 2008, pp. 99-116. 
[11] R. Buckingham and S. Venakides, Long-Time Asymptotics of the Nonlinear Schrödinger Equation Shock Problem, Comm. Pure Appl. Math. 60 (2007), no. 9, 1349-1414.

[12] P. Deift and X. Zhou, A steepest descent method for oscillatory Riemann-Hilbert problems. Asymptotics for the MKdV equation, Ann. of Math. (2) 137 (1993), no. 2, 295-368.

[13] P. A. Deift, A. R. Its, and X. Zhou, Long-time asymptotics for integrable nonlinear wave equations, Important developments in soliton theory, Springer Ser. Nonlinear Dynam., Springer, Berlin, 1993, pp. 181-204.

[14] A. S. Fokas, A unified transform method for solving linear and certain nonlinear PDEs, Proc. Roy. Soc. London Ser. A 453 (1997), no. 1962, 1411-1443.

[15] _ Integrable nonlinear evolution equations on the half-line, Comm. Math. Phys. 230 (2002), no. 1, $1-39$.

[16] A. S. Fokas, A. R. Its, and L.-Y. Sung, The nonlinear Schrödinger equation on the half-line, Nonlinearity 18 (2005), no. 4, 1771-1822.

[17] J Holmer, The initial-boundary-value problem for the $1 D$ nonlinear Schrödinger equation on the half-line, Differential Integral Equations 18 (2005), no. 6, 647-668.

[18] V. E Zakharov and A. B Shabat, Integration of nonlinear equations of mathematical physics by the inverse scattering method, Funk. Analiz Prilozh. 13 (1979), no. 3, 13-22.

[19] C. Zheng, Exact nonreflecting boundary conditions for one-dimensional cubic nonlinear Schrödinger equations, J. Comput. Phys. 215 (2006), no. 2, 552-565.

*imJ, Math., Université Paris Diderot Paris 7, 175 rue du Chevaleret, 75013 Paris, France E-mail address: aboutet@math.jussieu.fr

${ }^{\dagger}$ Math. Div., Inst. B. Verkin, 47 Lenin Avenue, 61103 Kharkiv, Ukraine

E-mail address: kotlyarov@ilt.kharkov.ua

E-mail address: shepelsky@yahoo.com

${ }^{\ddagger}$ Dept. Math. Sci., Tsinghua University, Beijing, 100084, P.R. China

E-mail address: czheng@math.tsinghua.edu.cn 